\title{
Association of Nuclear Factor Erythroid-2-Related Actor 2 Gene Polymorphisms with Diabetic Nephropathy in Chinese Patients
}

This article was published in the following Dove Press journal: International Journal of General Medicine

\author{
Jiancheng Wang \\ Meiling Yu \\ Jianrong Chen \\ Lingyan Zhu \\ Jianying Liu \\ Jixiong $\mathrm{Xu}$
}

Department of Endocrinology and Metabolism, First Affiliated Hospital of Nanchang University, Nanchang, Jiangxi, 330006, People's Republic of China

Correspondence: Jixiong Xu

Department of Endocrinology and Metabolism, First Affiliated Hospital of Nanchang University, No. 17, Yongwaizheng Street, Nanchang, Jiangxi, 330006, People's Republic of China

$\mathrm{Tel} / \mathrm{Fax}+86-79 \mathrm{I}-86836960$

Email Jixiong.Xu@ncu.edu.cn
Aim: Nuclear factor erythroid-2-related factor 2 (NRF2) has emerged as a therapeutic target in many diseases. To explore this further, we evaluated the relationships between the $-617 \mathrm{C} /$ A (rs6721961) polymorphisms within the NRF2 promoter and diabetic nephropathy (DN) in Chinese Han patients with type 2 diabetes mellitus (T2DM).

Methods: A total of 883 subjects with T2DM (500 without and 383 with DN) were enrolled in this study. Multivariable linear regression models were carried out to assess the association of DN with the -617C/A (rs6721961) polymorphisms.

Results: The AA genotype frequencies in patients with DN were significantly lower than those in patients without $\mathrm{DN}\left(\chi^{2}=8.04, p=0.018\right)$. Multivariate logistic regression analyses showed that individuals with the AA genotype had a significantly lower risk for DN (OR $0.52 ; 95 \%$ CI $0.28,0.94 ; p=0.029)$ than those with the CC genotype. Moreover, AA carriers had a significantly lower risk of DN (OR $0.46 ; 95 \%$ CI $0.26,0.82 ; p=0.009$ ) relative to those with the $\mathrm{CC}+\mathrm{CA}$ genotype, even after adjusting for known DN risk factors.

Conclusion: Our study indicated that the $-617 \mathrm{C} / \mathrm{A}$ polymorphism within the NRF2 promoter was significantly associated with DN in Chinese Han patients with T2DM.

Keywords: NRF2, polymorphism, diabetic nephropathy, Chinese population

\section{Introduction}

Diabetic nephropathy (DN) is one of the microvascular complications of diabetes and is the most common cause of end-stage renal disease (ESRD), and the cost for the treatment of diabetic patients with ESRD is increasing. ${ }^{1}$ Statistically, approximately $35 \%$ of people with diabetes will develop evidence of DN, and a substantial number of them would require dialysis. ${ }^{2}$ Therefore, an improved understanding of the factors involved in the onset and progression of $\mathrm{DN}$ is urgently required. To date, the major risk factors leading to DN include hyperglycemia, disorder of the rennin-angiotensin system, oxygen stress, inflammatory reaction and gene polymorphism. ${ }^{3}$ Recent evidence also suggests that nuclear factor erythroid2-related factor 2 (NRF2) plays an important role in the development of type 2 diabetes mellitus (T2DM) as well as its complications. ${ }^{4}$

The master antioxidant regulator NRF2 is a member of the cap-n-collar basic leucine zipper family that regulates the expression of many antioxidant genes in response to oxidative stress. ${ }^{5,6}$ Under physiological conditions, Kelch-like ECH associating protein 1 (Keap1) combines with NRF2, triggering its proteasomal degradation. ${ }^{7}$ In the presence of 
oxidative stress, NRF2 escapes such degradation and undergoes translocation to the nucleus, where it binds to antioxidant responsive elements (AREs) and upregulates the expression of various downstream genes encoding antioxidant enzymes, including heme oxygenase-1, NAD(P)H quinine oxidoreductase, superoxide dismutase 3 , and peroxiredoxin 1 , to avoid oxidative damage. $^{8-10}$ To date, NRF2 has emerged as a potential therapeutic strategy for various diseases because it can induce antioxidant enzymes and other cytoprotective enzymes. $^{4,11-13}$ A few single nucleotide polymorphisms (SNPs) have been identified in the NRF2 gene, such as rs264723, rs13001694, rs10497511, rs1806649. ${ }^{4}$ In particular, SNP -617C/A (rs6721961), a variant of the NRF2 gene in the upstream promoter region, is considered to play a pivotal role in the positive feedback loop of transcriptional activation of the NRF2 gene to regulate the NRF2 protein level. ${ }^{14}$

The $-617 \mathrm{C} / \mathrm{A}$ polymorphism has been gaining attention in the field of pharmacology and especially in the chemoprevention of diseases such as cancer and metabolic and cardiovascular diseases. ${ }^{15-18}$ A polymorphism in the NRF2 promoter ( $-617 \mathrm{C} / \mathrm{A})$ diminished the promoter activity compared with the wild type $(-617 \mathrm{CC})$ and was associated with a higher risk for developing acute lung injury, a disease related to reduced antioxidative cell defense. ${ }^{19}$ Furthermore, the rs6721961 polymorphism has been associated with oxidative stress and the risk of newly diagnosed T2DM. ${ }^{16}$ However, the role of the $-617 \mathrm{C} / \mathrm{A}$ (rs6721961) polymorphism within NRF2 in the regulation of DN in humans has never been investigated.

In this study, therefore, we evaluated the potential associations of the $-617 \mathrm{C} / \mathrm{A}$ (rs6721961) polymorphism with $\mathrm{DN}$ in a relatively large Chinese population.

\section{Patients and Methods Study Population}

This cross-sectional genotyping study enrolled consecutive Han Chinese patients with T2DM in the Department of Endocrinology and Metabolism, First Affiliated Hospital of Nanchang University, Nanchang, Jiangxi Province, China, between June 2011 and March 2015. Family trees were used to ensure their Han Chinese ethnic background. T2DM was diagnosed according to the 2003 American Diabetes Association diagnostic criteria for diabetes, based on a fasting blood glucose $(\mathrm{FBG})>7 \mathrm{mmol} / \mathrm{l}$, a causal blood glucose $>11.1 \mathrm{mmol} / 1$, or a postprandial 2-h blood glucose $(2 \mathrm{hBG})>11.1 \mathrm{mmol} / 1$ following a $75-\mathrm{g}$ oral glucose tolerance test, or a history of therapy for diabetes. Subjects were divided into two groups: without diabetic nephropathy $(\mathrm{n}=$ $500)$ and with diabetic nephropathy $(\mathrm{n}=383)$ according to their 24 hours microalbuminuria (MAU) and estimated glomerular filtration rate (eGFR). The patients without diabetic nephropathy who had at least 5 years of known duration of diabetes had no albuminuria (MAU $<30 \mathrm{mg} / 24 \mathrm{~h}$ ) and an eGFR $>60 \mathrm{~mL} \mathrm{~min}^{-1} 1.73 \mathrm{~m}^{-2}$ and were not receiving antihypertension treatment. The patients with diabetic nephropathy had overt albuminuria (MAU $>300 \mathrm{mg} / 24 \mathrm{~h}$ ) or eGFR $<60 \mathrm{~mL} \mathrm{~min}{ }^{-1} 1.73 \mathrm{~m}^{-2}$ (no end-stage renal disease or kidney transplantation), without any clinical or laboratory evidence of other kidney diseases. All patients underwent a complete eye examination that included dilated retinal examination and fundus photography or fundus fluorescein angiography. Diabetic retinopathy was evaluated by an experienced ophthalmologist. This study was approved by the ethics committee of the First Affiliated Hospital of Nanchang University (no. CDYFY2014009) and written informed consent was obtained from all individuals.

\section{Biochemical Analyses}

All of the patients underwent a standardized clinical and laboratory evaluation. Fasting blood samples were taken for measurement of FBG, hemoglobin A1c (HbA1c), total cholesterol (TC), low density lipoprotein cholesterol (LDL-C), high density lipoprotein (HDL-C), total triglyceride (TG), blood urea nitrogen (BUN), and serum creatinine. FBG, TC, HDL-C, LDL-C, TG, and serum creatinine were analyzed using the automated Olympus AU5421 chemistry analyzer (Olympus, Shizuoka, Japan). HbA1c content was measured using a Bio-Rad D-10 glycated hemoglobin analyzer (BioRad, Hercules, USA). Consider eGFR (mL $\mathrm{min}^{-1}$ $\left.1.73 \mathrm{~m}^{-2}\right)=175 \times\left[\right.$ serum creatinine $(\mathrm{mg} / \mathrm{dL})^{-1.234} \times$ age $\left.(\text { years })^{-0.179}\right] \times(0.79$, if female $) .^{20}$

\section{Genotyping}

For genotype analysis, genomic DNA was extracted from peripheral blood leukocytes of each individual using a DNA isolation kit (Bioteke, Beijing, China). The genomic DNA was subjected to polymerase chain reaction (PCR) with the following primers: Primer-F: 5'-GGTTCCCGTTTTTCTCCC AGCTCT-3', Primer-R: 5'-GGGTGGGGGGGGCTAAAG ATTT-3'. PCR was performed in a $20 \mu \mathrm{L}$ reaction volume containing $150 \mathrm{ng}$ of template DNA, $0.2 \mu \mathrm{mol} / \mathrm{L}$ of forward and reverse primers, $0.225 \mathrm{U}$ Taq DNA polymerase, 200 $\mu \mathrm{mol} / \mathrm{L}$ of each deoxynucleotide phosphate, and $2.0 \mathrm{mmol} / \mathrm{L}$ $\mathrm{Mg}^{2+}$ on the ABI 7300 PCR system (Applied Biosystems, Foster City, CA, USA). The amplification protocol consisted 
of $94^{\circ} \mathrm{C}$ for $5 \mathrm{~min}$, followed by 35 cycles of denaturation at $94^{\circ}$ $\mathrm{C}$ for $20 \mathrm{~s}$, annealing at $58^{\circ} \mathrm{C}$ for $30 \mathrm{~s}$, and extension at $72^{\circ} \mathrm{C}$ for $30 \mathrm{~s}$, with a final extension at $72^{\circ} \mathrm{C}$ for $5 \mathrm{~min}$. The PCR products were randomly selected for DNA sequencing analysis by Nanjing GenScript Biotech Corporation.

\section{Statistical Analyses}

Continuous variables were analyzed using a $t$-test and are presented as the mean \pm standard deviation (SD). For categorical variables, a chi-square test or Fischer exact test was used, and data are presented as percentages. Categorical variables and Hardy-Weinberg equilibrium were analyzed using a chisquare test. Intergroup comparisons of genotype and allele distribution were analyzed using Fisher's exact test and chisquare test, respectively. A two-sided $p$ value $<0.05$ was considered statistically significant.

Multivariable linear regression models were carried out to assess the association of diabetes events with the specific polymorphism. Odds ratios (OR) and $95 \%$ confidence intervals $(95 \% \mathrm{CI})$ were adjusted for known risk factors for age, sex, body mass index (BMI), SBP, DBP, FBG, HbAlc, TC, LDL-C, HDL-C, TG. Statistical analyses were performed using R software (http://www.R-project.org).

\section{Results}

\section{Clinical and Laboratory Characteristics of} Patients with T2DM

A total of 883 subjects with T2DM were enrolled in the study, including 383 patients in the DN group and 500 in the without DN group. The demographic and clinical characteristics for the DN and without DN groups are shown in Table 1. Participants with DN had a higher age, male gender, SBP, proportion of retinopathy, and levels of BUN, microalbuminuria, and serum creatinine than the without DN subjects. The frequencies of the AA genotype were significantly lower in patients with DN than in the without DN subjects. There were no significant differences in $\mathrm{HbA1c}$ percentages, FBG, BMI, DBP, TC, triglyceride, LDL-C, or HDL-C between the two groups.

\section{Clinical and Laboratory Characteristics of Patients with Type-2 Diabetic Nephropathy Classified According to $-617 \mathrm{C} / \mathrm{A}$ \\ Genotype}

As shown in Table 2, the levels of triglyceride in the AA genotype was significantly higher than that in the patients

Table I Clinical and Laboratory Characteristics of Patients with Type 2 Diabetes with and without Diabetic Nephropathy

\begin{tabular}{|c|c|c|c|c|}
\hline & $\begin{array}{c}\text { Total } \\
(n=883)\end{array}$ & $\begin{array}{l}\text { Without Diabetic Nephropathy } \\
\qquad(n=500)\end{array}$ & $\begin{array}{l}\text { With Diabetic Nephropathy } \\
\qquad(n=383)\end{array}$ & $P$ value* \\
\hline Male, No.(\%) & $462(52.3)$ & $242(48.4)$ & $220(57.4)$ & 0.008 \\
\hline Age, year & $60.6(11.0)$ & $59.4(10.6)$ & $62.1(11.4)$ & $<0.001$ \\
\hline Body mass index, $\mathrm{kg} / \mathrm{m}^{2}$ & $23.9(3.5)$ & $23.9(3.7)$ & $23.9(3.3)$ & 0.868 \\
\hline Nrf2 polymorphisms, No.(\%) & & & & 0.018 \\
\hline $\mathrm{CC}$ & $383(43.4)$ & $222(44.4)$ & $161(42.0)$ & \\
\hline $\mathrm{CA}$ & $434(49.2)$ & $231(46.2)$ & $203(53.0)$ & \\
\hline AA & $66(7.5)$ & $47(9.4)$ & $19(5.0)$ & \\
\hline Retinopathy, No.(\%) & $245(32.2)$ & $110(25.9)$ & $135(40.1)$ & $<0.001$ \\
\hline \multicolumn{5}{|l|}{ Blood pressure, $\mathrm{mmHg}$} \\
\hline Systolic BP & I35.4 (I8.6) & |32.8 (|8.1) & I38.8 (I8.7) & $<0.001$ \\
\hline Diastolic BP & $82.0(11.5)$ & $81.6(11.6)$ & $82.4(11.3)$ & 0.303 \\
\hline \multicolumn{5}{|l|}{ Laboratory results } \\
\hline $\mathrm{FBG}, \mathrm{mmol} / \mathrm{L}$ & $8.5(3.9)$ & $8.4(3.7)$ & $8.7(4.3)$ & 0.173 \\
\hline Hemoglobin Alc (\%) & $8.7(2.4)$ & $8.6(2.4)$ & $8.8(2.4)$ & 0.123 \\
\hline Total cholesterol, $\mathrm{mmol} / \mathrm{L}$ & $4.8(I I .7)$ & $5.1(15.4)$ & $4.5(1.3)$ & 0.454 \\
\hline Triglyceride, $\mathrm{mmol} / \mathrm{L}$ & $1.6(1.7)$ & $1.5(1.8)$ & $1.7(\mathrm{I} .5)$ & 0.106 \\
\hline $\mathrm{HDL}-\mathrm{C}, \mathrm{mmol} / \mathrm{L}$ & I.3 (3.7) & I.4 (4.8) & $1.2(1.2)$ & 0.481 \\
\hline LDL-C, $\mathrm{mmol} / \mathrm{L}$ & $3.0(7.8)$ & $2.6(0.9)$ & $3.6(I I .8)$ & 0.054 \\
\hline BUN, mmol/L & $6.9(5.2)$ & $5.4(1.9)$ & $8.9(7.1)$ & $<0.001$ \\
\hline Microalbuminuria, mg/24h & $42.6(54.5)$ & $6.7(6.0)$ & $87.5(54.9)$ & $<0.001$ \\
\hline Serum creatinine, $\mu \mathrm{mol} / \mathrm{L}$ & $87.4(76.6)$ & $60.1(16.0)$ & $122.5(104.4)$ & $<0.001$ \\
\hline
\end{tabular}

Notes: Data are the mean $\pm \mathrm{SD}$, or $\mathrm{n}(\%)$. *P values were obtained by an unpaired Student's $t$-test analysis or $\chi^{2}$ test, as appropriate.

Abbreviations: FBG, fasting blood glucose; HDL-C, high density lipoprotein; LDL-C, low density lipoprotein cholesterol; BUN, blood urea nitrogen. 
Table 2 Clinical and Laboratory Characteristics of Patients with Type-2 Diabetic Nephropathy Classified According to Their -6I7C/A Genotypes

\begin{tabular}{|c|c|c|c|c|}
\hline & $C C(n=161)$ & CA $(n=203)$ & AA $(n=19)$ & $P$ value* \\
\hline Male, No.(\%) & $103(64.0)$ & $22 I(52.2)$ & II (57.9) & 0.079 \\
\hline Age, year & $61.3(11.1)$ & $62.6(11.8)$ & $63.5(9.0)$ & 0.451 \\
\hline Body mass index, $\mathrm{kg} / \mathrm{m}^{2}$ & $23.8(3.3)$ & $24.0(3.4)$ & $23.9(3.3)$ & 0.920 \\
\hline Retinopathy, No.(\%) & $64(42.4)$ & $65(38.9)$ & $6(31.6)$ & 0.607 \\
\hline \multicolumn{5}{|l|}{ Blood pressure, $\mathrm{mmHg}$} \\
\hline Systolic BP & I38.0 (19.5) & I39.5 (|8.6) & | $38.4(\mid 3.6)$ & 0.747 \\
\hline Diastolic BP & $83.0(12.0)$ & $82.0(11.1)$ & $83.2(8.3)$ & 0.676 \\
\hline \multicolumn{5}{|l|}{ Laboratory results } \\
\hline $\mathrm{FBG}, \mathrm{mmol} / \mathrm{L}$ & $9.1(4.7)$ & $8.6(3.9)$ & $6.9(3.4)$ & 0.092 \\
\hline Hemoglobin Alc (\%) & $8.8(2.4)$ & $8.7(2.4)$ & $9.3(2.2)$ & 0.649 \\
\hline Total cholesterol, $\mathrm{mmol} / \mathrm{L}$ & $4.6(1.5)$ & $4.5(1.2)$ & 4.I (I.I) & 0.294 \\
\hline Triglyceride, $\mathrm{mmol} / \mathrm{L}$ & I.8 (I.5) & I.5 (I.0) & $2.5(3.5)$ & 0.008 \\
\hline $\mathrm{HDL}-\mathrm{C}, \mathrm{mmol} / \mathrm{L}$ & I.I (0.4) & $1.3(1.6)$ & I.I (0.4) & 0.400 \\
\hline LDL-C, $\mathrm{mmol} / \mathrm{L}$ & $4.8(18.1)$ & $2.8(1.1)$ & $2.5(1.0)$ & 0.239 \\
\hline $\mathrm{BUN}, \mathrm{mmol} / \mathrm{L}$ & $9.3(7.6)$ & $8.8(7.0)$ & $6.8(2.6)$ & 0.343 \\
\hline Microalbuminuria, mg/24h & $86.1(54.7)$ & $88.2(54.6)$ & $91.5(62.9)$ & 0.905 \\
\hline Serum creatinine, $\mu \mathrm{mol} / \mathrm{L}$ & | $31.4(\mid 19.0)$ & II $5.0(94.2)$ & I27.2 (67.4) & 0.328 \\
\hline
\end{tabular}

Notes: Data are the mean \pm SD, or $n(\%)$. *P values were obtained by an unpaired Student's $t$-test analysis or $\chi^{2}$ test, as appropriate.

Abbreviations: FBG, fasting blood glucose; HDL-C, high density lipoprotein; LDL-C, low density lipoprotein cholesterol; BUN, blood urea nitrogen.

with the $\mathrm{CC}$ or CA genotype. There were no significant differences in other clinical and laboratory characteristics between the genotype groups.

\section{Association of DN with $-617 \mathrm{C} / \mathrm{A}$ Polymorphism in Patients with T2DM}

As shown in Table 3, the genotype frequencies of the $-617 \mathrm{C} / \mathrm{A}$ polymorphism in the control group were in Hardy-Weinberg equilibrium $\left(\chi^{2}=1.40, p=0.236\right)$. There were significant differences in the allelic frequency of the $-617 \mathrm{C} / \mathrm{A}$ polymorphism between $\mathrm{DN}$ and without DN. The frequencies of the CC, CA, and AA genotypes were $44.4,46.2$, and $9.4 \%$, respectively, in the group

Table 3 The Genotypic Distribution of the $-617 \mathrm{C} / \mathrm{A}$ Polymorphism in Type 2 Diabetic Patients with and without Diabetic Nephropathy

\begin{tabular}{|l|c|c|c|c|}
\hline & $\begin{array}{c}\text { Without } \\
\text { Diabetic } \\
\text { Nephropathy } \\
\text { n (\%) }\end{array}$ & $\begin{array}{c}\text { With } \\
\text { Diabetic } \\
\text { Nephropathy } \\
\text { n (\%) }\end{array}$ & $\chi^{2}$ & P value* \\
\hline Genotype & $222(44.4)$ & $161(42.0)$ & 8.04 & 0.018 \\
CC & $231(46.2)$ & $203(53.0)$ & & \\
CA & $47(9.4)$ & $19(5.0)$ & & \\
AA & & & \\
\hline
\end{tabular}

Note: ${ }^{*} P$ values were obtained by an $\chi^{2}$ test. without $\mathrm{DN}$ and 42.0, 53.0, and 5.0\%, respectively, in the group with $\mathrm{DN}\left(\chi^{2}=8.04, p=0.018\right)$.

Individuals carrying with the AA genotype had a significantly lower risk for DN (OR 0.52; 95\% CI 0.28, $0.94 ; p=0.029)$ than those with the $\mathrm{CC}$ genotype; Compared to those with the $\mathrm{CC}+\mathrm{CA}$ genotype, AA carriers had a significantly lower risk of DN (OR $0.46 ; 95 \%$ CI 0.26 , $0.82 ; p=0.009$ ) after adjusting for age, sex, BMI, SBP, DBP, FBG, HbA1c, TC, LDL-C, HDL-C, TG. (Table 4).

\section{Stratified Analyses by Important Covariables}

Stratified analyses were performed to further assess the association between -617C/A polymorphism and DN in age and sex subgroups. None of the following variables, including age ( $<60$ vs $\geq 60$ years; $P$ for interaction $=0.558$ ), sex $(P$ for interaction $=0.653$ ) significantly modified the association between $-617 \mathrm{C} / \mathrm{A}$ polymorphism and DN (Table 5).

\section{Discussion}

NRF2 is a key regulator of DN through the suppression of oxidative stress assault, ${ }^{21}$ which is a major contributor to the development and progression of DN. To our knowledge, this is the first report providing clinical evidence that the $617 \mathrm{C} / \mathrm{A}$ SNP, which is located in the promoter region of the NRF2 gene, is associated with DN in Chinese 
Table 4 Univariate and Multivariate Odds Ratios of Diabetic Nephropathy According to the -617C/A Polymorphism

\begin{tabular}{|l|c|c|c|c|}
\hline \multirow{2}{*}{} & No. of Cases/Control Subjects & \multicolumn{2}{|c|}{ OR (95\% CI) } & \multirow{2}{*}{ P value* } \\
\cline { 2 - 4 } & & Unadjusted & & \\
\hline Genotype & $161 / 222$ & & 1.00 & \\
CC & $203 / 231$ & 1.00 & $1.23(0.92,1.64)$ & 0.162 \\
CA & $19 / 47$ & $1.21(0.92,1.60)$ & $0.52(0.28,0.94)$ & 0.029 \\
AA & $364 / 453$ & $0.56(0.32,0.99)$ & 1.00 & 0.009 \\
CC+CA & $19 / 47$ & $0.50(0.29,0.87)$ & $0.46(0.26,0.82)$ & 0 \\
AA & & 1.00 & \\
\hline
\end{tabular}

Notes: *Adjusted for age, sex, body mass index, systolic blood pressure, diastolic blood pressure, fasting blood glucose, hemoglobin Alc, total cholesterol, low density lipoprotein cholesterol, high density lipoprotein, total triglyceride.

Table 5 Subgroup Analysis of Diabetic Nephropathy According to the -6I7C/A Polymorphism

\begin{tabular}{|c|c|c|c|c|}
\hline \multirow[t]{2}{*}{ Source } & \multicolumn{2}{|c|}{ OR $(95 \% \mathrm{CI})$} & \multirow[t]{2}{*}{ P value* } & \multirow[t]{2}{*}{ P for Interaction* } \\
\hline & $\mathrm{CC}+\mathrm{CA}$ & AA & & \\
\hline Age, years & & & & 0.558 \\
\hline$<60$ & 1.00 & $0.58(0.25, \mathrm{I} .37)$ & 0.215 & \\
\hline$\geq 60$ & 1.00 & $0.36(0.16,0.83)$ & 0.016 & \\
\hline Sex & & & & 0.653 \\
\hline Male & 1.00 & $0.5 \mathrm{I}(0.23, \mathrm{I} .14)$ & 0.107 & \\
\hline Female & 1.00 & $0.38(0.16,0.93)$ & 0.034 & \\
\hline
\end{tabular}

Notes: *Adjusted for age, sex, body mass index, systolic blood pressure, diastolic blood pressure, fasting blood glucose, hemoglobin Alc, total cholesterol, low density lipoprotein cholesterol, high density lipoprotein, total triglyceride.

patients. Individuals with AA homozygotes had a significantly lower risk of DN than those with $\mathrm{CC}$ homozygotes, even after adjusting for known DN risk factors.

Previous research has paid little attention to the association between NRF2 single nucleotide polymorphisms and $\mathrm{DN}$, and there are only limited data and disputable results linking the NRF2 gene with diabetes. In line with our findings, a study conducted in Mexico showed that the rs6721961 (-617C/A) polymorphism of the NRF2 gene was associated with diabetes in male subjects, AA carriers had lower glucose concentrations, and the OR revealed that A carriers had a lower risk of developing diabetes. ${ }^{15}$ However, Wang et al observed that AA carriers in the NRF2 rs6721961 polymorphism had lower total antioxidative capacity, superoxide dismutase, catalase, glutathione, and glutathione peroxidase activity and had a significantly higher risk of developing T2DM (OR $1.56 ; 95 \%$ CI $1.11,2.20 ; \mathrm{p}=0.011)$ than those with the CC genotype. ${ }^{16}$ In the previous two studies, ${ }^{15,16}$ they evaluated the rs6721961 (-617C/A) polymorphism with T2DM versus the $-617 \mathrm{C} / \mathrm{A}$ polymorphism with microvascular complication of T2DM, which was assessed in the present study. Additionally, the included population and other characteristics, such as the patients who were selected and the sample size, might explain the inconsistent results, but we believe that the NRF2 rs6721961 polymorphism is worthy of further examination.

There are some reports linking NRF2 genetic variations and metabolic disease in different racial populations. Polymorphisms in the promoter region of NRF2 have been previously reported in Japanese hemodialysis patients. It was found that the mutant allele of rs6721961 ( $-617 \mathrm{C} / \mathrm{A})$ is significantly associated with systolic and diastolic blood pressure. ${ }^{17}$ In a study including white and African American populations, $-617 \mathrm{~A}$ variant allele carriers had significantly higher forearm vascular resistance at baseline compared with wild-type individuals in whites. ${ }^{22}$ One study observed that T2DM patients with complications had a higher frequency of four mutant NRF2 single nucleotide polymorphisms (rs2364723 C allele, rs10497511 G allele, rs1962142 A allele and rs6726395 G allele) than T2DM patients without complications in a cohort of Chinese participants. ${ }^{4}$

Oxidative stress is emerging as an important contributing factor of DN pathology and progression.,23 NRF2 is a pivotal transcription factor and functions to 
upregulate the expression of antioxidant genes in response to oxidative stress. In a mouse model of diabetes induced by methylglyoxal, treatment with resveratrol, which has been proposed as an effective treatment that helps lower the risk of developing complications of diabetes, markedly improved blood glucose levels based on the oral glucose tolerance test and promoted NRF2 phosphorylation of the pancreas. ${ }^{24}$ Accordingly, the treatment of normal human renal epithelial HK-2 cells with NRF2 inducers effectively elevated the expression of aldo-keto reductase levels. ${ }^{25}$ Targeted disruption of NRF2 significantly reduced antioxidant capacity in mice and thus increased high-level susceptibility to a variety of toxic chemicals and carcinogens. ${ }^{26,27}$ Thus, lower antioxidant capacity due to lower expression of the NRF2 gene is a risk factor for complications of diabetes. The findings of the present study revealed that high activity of NRF2, as shown in AA allele carriers of the $-617 \mathrm{C} / \mathrm{A}$ SNP, seems to be associated with a low risk of DN. This indicates that the AA genotype plays a prominent role in the renal protection of humans against oxidative stress. However, a more complete understanding of the mechanisms by which NRF2 protects against DN is needed.

There are some limitations in this study. First, we were not able to measure plasma reactive oxygen species concentrations in all of the involved participants, particularly in DN subjects. Second, all of the participants in our study were Han Chinese. Whether the results can be extrapolated to other populations requires further verification. Third, we analyzed only one SNP, rs6721961, and haplotypes of the promoter region only, which does not exclude any association of other regions around or within the gene. Finally, the present findings obtained in a cross-sectional study are explorative in nature, and replication in independent prospective population-based studies with different ethnicities is needed to determine whether the $-617 \mathrm{C} / \mathrm{A}$ polymorphism influences patients with DN.

In conclusion, the present study indicated that the $-617 \mathrm{C} / \mathrm{A}$ polymorphism in the NRF2 gene was significantly associated with DN in Chinese Han patients with T2DM. Further studies are necessary to validate the impact of the polymorphism in the NRF2 gene on oxidative stress and the risk of T2DM and its complications.

\section{Author Contributions}

All authors made substantial contributions to conception and design, acquisition of data, or analysis and interpretation of data; took part in drafting the article or revising it critically for important intellectual content; agreed to submit to the current journal; gave final approval of the version to be published; and agree to be accountable for all aspects of the work.

\section{Funding}

The study was supported by funding from the following: the National Natural Science Funds of China (Nos. 81760168 and 81460018) and the National Natural Science Funds of Jiangxi Province (No. 20192B AB205031).

\section{Disclosure}

All authors declare they do not have conflict of interests.

\section{References}

1. Fox CS, Matsushita K, Woodward M, et al. Associations of kidney disease measures with mortality and end-stage renal disease in individuals with and without diabetes: a meta-analysis. Lancet. 2012;380 (9854):1662-1673. doi:10.1016/S0140-6736(12)61350-6

2. Adler A, Casula A, Steenkamp R, et al. Association between glycemia and mortality in diabetic individuals on renal replacement therapy in the U.K. Diabetes Care. 2014;37(5):1304-1311. doi:10.2337/dc13-0553

3. Zheng S, Powell DW, Zheng F, et al. Diabetic nephropathy: proteinuria, inflammation, and fibrosis. J Diabetes Res. 2016;2016:5241549.

4. Xu X, Sun J, Chang X, et al. Genetic variants of nuclear factor erythroid-derived 2-like 2 associated with the complications in Han descents with type 2 diabetes mellitus of Northeast China. J Cell Mol Med. 2016;20(11):2078-2088. doi:10.1111/jcmm.12900

5. Moi P, Chan K, Asunis I, et al. Isolation of NF-E2-related factor 2 (Nrf2), a NF-E2-like basic leucine zipper transcriptional activator that binds to the tandem NF-E2/AP1 repeat of the beta-globin locus control region. Proc Natl Acad Sci USA. 1994;91(21):9926-9930. doi:10.1073/pnas.91.21.9926

6. Cho HY. Genomic structure and variation of nuclear factor (erythroid-derived 2)-like 2. Oxid Med Cell Longev. 2013;2013:286524. doi:10.1155/2013/286524

7. Canning P, Sorrell FJ, Bullock AN. Structural basis of Keap1 interactions with Nrf2. Free Radic Biol Med. 2015;88(Pt B):101-107. doi:10.1016/j.freeradbiomed.2015.05.034

8. Zenkov NK, Menshchikova EB, Tkachev VO. Keap1/Nrf2/ARE redox-sensitive signaling system as a pharmacological target. Biochemistry (Mosc). 2013;78(1):19-36. doi:10.1134/S000629791 3010033

9. Jaiswal AK. Nrf2 signaling in coordinated activation of antioxidant gene expression. Free Radic Biol Med. 2004;36(10):1199-1207. doi:10.1016/j.freeradbiomed.2004.02.074

10. Itoh K, Tong KI, Yamamoto M. Molecular mechanism activating nrf2-keap1 pathway in regulation of adaptive response to electrophiles. Free Radic Biol Med. 2004;36(10):1208-1213. doi:10.1016/j. freeradbiomed.2004.02.075

11. Ruiz S, Pergola PE, Zager RA, et al. Targeting the transcription factor $\mathrm{Nrf} 2$ to ameliorate oxidative stress and inflammation in chronic kidney disease. Kidney Int. 2013;83(6):1029-1041. doi:10.1038/ ki.2012.439

12. Korytina GF, Akhmadishina LZ, Aznabaeva YG, et al. Associations of the NRF2/KEAP1 pathway and antioxidant defense gene polymorphisms with chronic obstructive pulmonary disease. Gene. 2019;692:102-112. doi:10.1016/j.gene.2018.12.061 
13. Chen S, Pan H, Chen Y, et al. Association between genetic polymorphisms of NRF2, KEAP1, MAFF, MAFK and anti-tuberculosis drug-induced liver injury: a nested case-control study. Sci Rep. 2019;9(1):14311. doi:10.1038/s41598-019-50706-y

14. Yamaguchi $\mathrm{Y}$, Kamai T, Higashi S, et al. Nrf2 gene mutation and single nucleotide polymorphism rs6721961 of the Nrf2 promoter region in renal cell cancer. BMC Cancer. 2019;19(1):1137. doi:10.1186/s12885-019-6347-0

15. Jiménez-Osorio AS, González-Reyes S, García-Niño WR, et al. Association of nuclear factor-erythroid 2-related factor 2, thioredoxin interacting protein, and heme oxygenase-1 gene polymorphisms with diabetes and obesity in mexican patients. Oxid Med Cell Longev. 2016;2016:7367641. doi:10.1155/2016/7367641

16. Wang X, Chen H, Liu J, et al. Association between the NF-E2 related factor 2 gene polymorphism and oxidative stress, anti-oxidative status, and newly-diagnosed type 2 diabetes mellitus in a chinese population. Int J Mol Sci. 2015;16(7):16483-16496. doi:10.3390/ ijms 160716483

17. Shimoyama Y, Mitsuda Y, Tsuruta Y, et al. Polymorphism of Nrf2, an antioxidative gene, is associated with blood pressure and cardiovascular mortality in hemodialysis patients. Int J Med Sci. 2014;11 (7):726-731. doi:10.7150/ijms.8590

18. Reszka E, Jablonowski Z, Wieczorek E, et al. Polymorphisms of NRF2 and NRF2 target genes in urinary bladder cancer patients. $J$ Cancer Res Clin Oncol. 2014;140(10):1723-1731. doi:10.1007/ s00432-014-1733-0

19. Marzec JM, Christie JD, Reddy SP, et al. Functional polymorphisms in the transcription factor NRF2 in humans increase the risk of acute lung injury. FASEB J. 2007;21(9):2237-2246. doi:10.1096/fj.067759 com
20. Zhang L, Wang F, Wang L, et al. Prevalence of chronic kidney disease in China: a cross-sectional survey. Lancet. 2012;379 (9818):815-822. doi:10.1016/S0140-6736(12)60033-6

21. Priestley JR, Kautenburg KE, Casati MC, et al. The NRF2 knockout rat: a new animal model to study endothelial dysfunction, oxidant stress, and microvascular rarefaction. Am J Physiol Heart Circ Physiol. 2016;310(4):H478-87. doi:10.1152/ajpheart.00586.2015

22. Marczak ED, Marzec J, Zeldin DC, et al. Polymorphisms in the transcription factor NRF2 and forearm vasodilator responses in humans. Pharmacogenet Genomics. 2012;22(8):620-628. doi:10.1 097/FPC.0b013e32835516e5

23. Jha JC, Banal C, Chow BS, et al. Diabetes and kidney disease: role of oxidative stress. Antioxid Redox Signal. 2016;25(12):657-684. doi:10.1089/ars.2016.6664

24. Cheng AS, Cheng YH, Lee CY, et al. Resveratrol protects against methylglyoxal-induced hyperglycemia and pancreatic damage in vivo. Nutrients. 2015;7(4):2850-2865. doi:10.3390/nu7042850

25. Jung KA, Choi BH, Nam CW, et al. Identification of aldo-keto reductases as NRF2-target marker genes in human cells. Toxicol Lett. 2013;218(1):39-49. doi:10.1016/j.toxlet.2012.12.026

26. Cho HY, Gladwell W, Yamamoto M, et al. Exacerbated airway toxicity of environmental oxidant ozone in mice deficient in Nrf2. Oxid Med Cell Longev. 2013;2013:254069. doi:10.1155/2013/254069

27. Aoki Y, Sato H, Nishimura N, et al. Accelerated DNA adduct formation in the lung of the Nrf2 knockout mouse exposed to diesel exhaust. Toxicol Appl Pharmacol. 2001;173(3):154-160. doi:10.1 006/taap.2001.9176
International Journal of General Medicine

\section{Publish your work in this journal}

The International Journal of General Medicine is an international, peer-reviewed open-access journal that focuses on general and internal medicine, pathogenesis, epidemiology, diagnosis, monitoring and treatment protocols. The journal is characterized by the rapid reporting of reviews, original research and clinical studies

\section{Dovepress}

across all disease areas. The manuscript management system is completely online and includes a very quick and fair peer-review system, which is all easy to use. Visit http://www.dovepress.com/ testimonials.php to read real quotes from published authors. 